\title{
EXPERIMENTAL AND NUMERICAL STUDY OF AN OBLIQUELY TOWED SHIP MODEL IN CONFINED WATERS
}

\author{
Momchil Terziev ${ }^{a, 1}$, Khaled Elsherbiny $^{b}$, Tahsin Tezdogan ${ }^{a}$, Atilla Incecik ${ }^{a}$ \\ aDepartment of Naval Architecture, Ocean and Marine Engineering, University of Strathclyde, Glasgow, UK \\ ${ }^{b}$ Arab Academy for Science, Technology and Maritime Transport, Alexandria, Egypt
}

\section{ABSTRACT}

In this study, the forces and moments acting on the KCS ship model as a result of oblique towing at 10 and 20 degrees drift angles are evaluated experimentally and numerically via a commercial Reynolds averaged Navier-Stokes solver. For the purposes of this work, the KCS hull is modelled both experimentally and numerically at a scale factor of 1:75. The adopted case-studies feature both horizontal and vertical restrictions. Thus, the subject of this work is the oblique motion of a ship in a narrow canal with a depth of $h / T=2.2$. The relative impact of turbulence modelling is assessed by comparing the computed integral quantities via several eddy-viscosity closure strategies. These include significant variants of the $k-\epsilon$ and $k-\omega$ models as well as a widely used one-equation closure. Multiphase numerical simulations are performed at several of the experimentally investigated depth Froude numbers for each drift angle condition in order to fully capture the physics of the problem at hand. The present study aims to provide a quantitative evaluation of the performance of the adopted turbulence models and recommended the best closure strategy for the class of investigated problems. towing.

Keywords: EFD, CFD, restricted waters, drift, oblique

\section{NOMENCLATURE}

$\begin{array}{ll}C_{M} & \text { Moment coefficient [-] } \\ C_{X} & \text { Resistance coefficient along the } x-\text { axis [-] } \\ C_{Y} & \text { Resistance coefficient along the } y-\text { axis [-] } \\ F_{T} & \text { Total resistance [N] } \\ F_{X} & x-\text { component of resistance [N] } \\ F_{Y} & y-\text { component of resistance [N] } \\ F_{h} & \text { Depth Froude number [-] } \\ i & \text { Number of experimental runs } \\ k & \text { Coverage factor }=1.96 \\ L & \text { Length between perpendiculars }=3.066 \mathrm{~m} \\ M & \text { Hydrodynamic moment [Nm] } \\ s & \text { Standard deviation of measurements } \\ \mathrm{S} & \text { Wetted surface area }=1.694 \mathrm{~m}^{2}\end{array}$

$\begin{array}{ll}u_{A} & \text { Type A uncertainty } \\ U_{A} & \text { Type A uncertainty with } 95 \% \text { confidence } \\ \beta & \text { Drift angle }\left[^{\circ}\right] \\ \rho & \text { Water density }=988.8 \mathrm{~kg} / \mathrm{m}^{3}\end{array}$

\section{INTRODUCTION}

In recent years, numerous studies examining the performance of ships in shallow waters have emerged. These examine in great detail every aspect of a ship's performance, ranging from shallow/restricted water effects on resistance, running sinkage and trim, propeller performance, etc. [1]-[8]. Several of these have researched the performance of a ship when sailing at a drift angle [9]-[12] in both deep and shallow waters. Such examinations are important, because a ship may experience large hydrodynamic forces and moments if sailing at an angle to the incident flow. A case where this occurs frequently is in shallow waters during maneuvering. Several studies have demonstrated that the proximity of the seabed has a pronounced effect on the hydrodynamic forces and moments acting on the hull [9], [10], [13]. These are of particular interest, because it is well-known that in shallow waters, maneuverability is compromised when compared to deep waters [14]. Therefore, it is warranted to investigate the hydrodynamic performance of a

\footnotetext{
${ }^{1}$ Contact author: momchil.terziev@strath.ac.uk
} 
ship in cases where the width of the seaway is restricted, as well as the depth. Conditions that may fit this description could be encountered when a ship is sailing in a canal or entering a port, for example.

The purpose of this study is twofold. Firstly, the experimental campaign of Elsherbiny et al. [15], [16], which focused on canals is extended to oblique towing. The KRISO container ship (KCS), at a scale factor of 1:75 is used, as in the aforementioned works. The specific cases this study focuses on are the oblique towing of the KCS at an angle of $10^{\circ}$ and $20^{\circ}$ in a canal of width, equal to $4.6 \mathrm{~m}$ and a depth of $0.32 \mathrm{~m}$ (i.e. a depthto-draught ratio of 2.2). The ship is towed at a variety of speeds to enable comparison with numerical predictions, which forms the second part of this study, where a Reynolds Averaged NavierStokes (RANS) solver (Star-CCM+) is used to re-create the test cases. Since turbulence and its modelling are known to play a critical role in the numerical prediction of ships towed at a drift angle, four different widely used approaches are compared. These comprise the two-layer realizable $k-\epsilon$ model [17] (with the coefficients of Launder and Sharma [18]), the $k-\omega$ model [19], the $k-\omega S S T$ (Shear Stress Transport) model [20], and the oneequation Spalart-Allmaras [21] model (referred to as 'SPAL' hereafter for brevity). Details of the implementation of these models can be accessed in the RANS software manual [22].

The present study is organized as follows. Section 1 contains a description of the experiment, as well as an estimate of the experimental uncertainty. Section 1 also summarizes the experimental data to facilitate other studies. Section 2 contains a brief description of the numerical implementation. This is followed by the results and discussion in Section 3. Finally, concluding remarks are given in Section 4.

\subsection{The experimental campaign}

In this experiment the sinkage, trim and resistance has been measured for $10^{\circ}$ and $20^{\circ}$ drift angles. This experimental work was conducted at the Kelvin Hydrodynamics Lab at the University of Strathclyde. The towing tank principle dimensions are shown in Table 1 and Figure 1. The maximum speed of the carriage is $5 \mathrm{~m} / \mathrm{s}$ which is driven along rails by a computercontrolled digital driven DC motor. The tank is equipped with a wave-maker able to generate regular and irregular waves up to $0.5 \mathrm{~m}$ height. The tank is also fitted with computer-controlled unit for variable water depth. The water depth at the tank was set at $0.32 \mathrm{~m}$ for all shallow water tests, giving a depth-to-draught ratio of 2.2.

Table 1: Main dimensions of the tank

\begin{tabular}{|l|l|}
\hline Total length & $76 \mathrm{~m}$ \\
\hline Width & $4.6 \mathrm{~m}$ \\
\hline Maximum water depth & $2.5 \mathrm{~m}$ \\
\hline
\end{tabular}

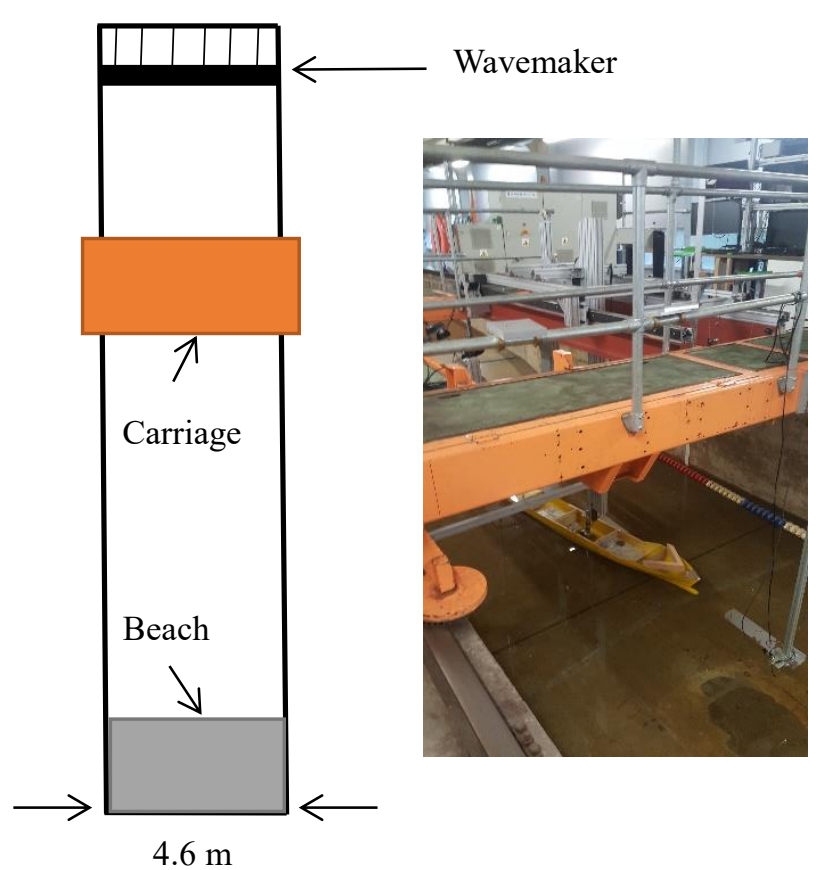

FIGURE 1: Towing tank facility

A KCS model was used to experimentally test the ship's characteristics at different speeds and different drift angles. The model was constructed as geometrically similar to the full-scale ship with a scale factor of $1: 75$. Full- and model-scale particulars of the ship are given in Table 2.

Table 2: Principal characteristics of the KCS

\begin{tabular}{|l|l|l|}
\hline Parameters & $\begin{array}{l}\text { Full- } \\
\text { scale }\end{array}$ & $\begin{array}{l}\text { Model- } \\
\text { scale }\end{array}$ \\
\hline Scale & 1.00 & 75 \\
\hline $\begin{array}{l}\text { Length between perpendiculars } \\
(\mathrm{m})\end{array}$ & 230 & 3.067 \\
\hline Length at water line (m) & 232.5 & 3.1 \\
\hline Breadth at water line (m) & 32.2 & 0.429 \\
\hline Depth (m) & 19 & 0.25 \\
\hline Draft (m) & 10.8 & 0.144 \\
\hline Displacement $\left(\mathrm{m}^{3}\right)$ & 52030 & 0.123 \\
\hline $\begin{array}{l}\text { Wetted surface area w/o rudder } \\
\left(\mathrm{m}^{2}\right)\end{array}$ & 9530 & 1.694 \\
\hline Block Coefficient & 0.651 & 0.651 \\
\hline
\end{tabular}

Three 3D motion cameras were employed to measure the sinkage and trim. The optical tracking systems for these cameras are infrared. Using data from the three cameras and the system are used to calculate the $3 \mathrm{D}$ positions of the markers, which are 
attached to a model. A dynamometer force measurement was used to measure the resistance. The dynamometer was attached at the midship point. The measured lift and drag force components on the model hull are used to compute the total resistance acting on the model as shown in Eq. (1) and Figure 2.

$$
F_{T}=F_{X} \cos \beta+F_{Y} \sin \beta
$$

where $F_{T}$ is total resistance acting on the model, $F_{X}$ is force measured along $x$-axis, $F_{Y}$ is the force measured along $y$-axis, and $\beta$ is the drift angle. The forces are non-dimensionalised via division by $0.5 \rho U^{2} S$, whereas the hydrodynamic moment: $0.5 \rho U^{2} L^{3}$, following Longo and Stern [23]. Here, $U$ is the ship speed, $\rho=988.8 \mathrm{~kg} / \mathrm{m}^{3}$ is the water density, and $\mathrm{S}$ is the ship's underwater area. Thus, the total force $\left(\mathrm{F}_{\mathrm{T}}\right)$, drag force $\left(\mathrm{F}_{\mathrm{X}}\right)$, lift force $\left(\mathrm{F}_{\mathrm{Y}}\right)$, and moment $(\mathrm{M})$ become $\mathrm{C}_{\mathrm{T}}, \mathrm{C}_{\mathrm{X}}, \mathrm{C}_{\mathrm{Y}}$, and $\mathrm{C}_{\mathrm{M}}$, respectively.

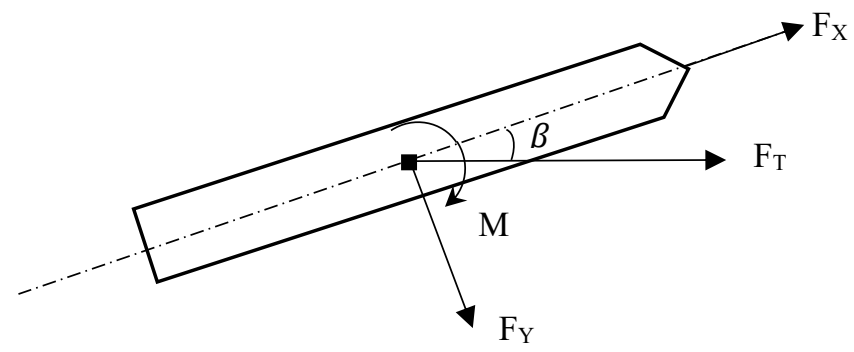

FIGURE 2: Definition of the forces and moments, acting on the ship. Here, $\mathrm{M}$ is the hydrodynamic moment.

The test were performed in fresh water and the water temperature was recorded regularly during the tests. Model drag force, lift force, dynamic trim, sinkage and actual speed of the model were recorded during the runs for calm water.

\subsection{Experimental uncertainty}

Estimation of experimental uncertainty is needed to assess the confidence in the presented results. The uncertainty is divided into two types $\mathrm{A}$ and $\mathrm{B}$, based on the way that the uncertainty is evaluated [24]. Type A standard uncertainty, also termed 'random uncertainty' [25], is a method of determining the standard uncertainty by conducting an evaluation of a statistical analysis of a series of repeated observations. The experiments were carried out by repeating the test five times in the same conditions at the depth Froude number $\left(\mathrm{F}_{\mathrm{h}}\right)$ equal to 0.335. Eq. (2) shows how to measure the uncertainty using the Type A $\left(u_{A}\right)$ method:

$u_{A}=\sqrt{\frac{s^{2}}{i}}$

where $i$ is the number of repeated observations and $s$ is the standard deviation of the values. To obtain a $95 \%$ level of confidence uncertainty, the uncertainty type $\mathrm{A}\left(u_{A}\right)$ is multiplied by a coverage factor $k$ as shown Eq. (3).
$U_{A}=k u_{A}$

where $k=1.96$ for a $95 \%$ level of confidence [25]. The results from the uncertainty analysis are shown in Table 3.

Table 3: Uncertainty of repeat measurements

\begin{tabular}{|l|l|l|l|}
\hline $\mathrm{F}_{\mathrm{h}}$ & $\begin{array}{l}\mathrm{U}_{\mathrm{A}} \text { for } \\
\text { resistance }(\mathrm{N})\end{array}$ & $\begin{array}{l}\mathrm{U}_{\mathrm{A}} \text { for } \\
\text { sinkage } \\
(\mathrm{mm})\end{array}$ & $\begin{array}{l}\mathrm{U}_{\mathrm{A}} \text { for trim } \\
(\mathrm{deg} .)\end{array}$ \\
\hline 0.335 & 0.05 & 0.0517 & 0.0047 \\
\hline Percentage & $1.1 \%$ & $2.3 \%$ & $0.96 \%$ \\
\hline
\end{tabular}

Type B standard uncertainty or otherwise termed 'systematic uncertainty' [25] is a method of standard uncertainty estimation obtained by means, other than statistical analysis, for example instrument calibration data and linear regression analyses. In the present work all drift angle tests were carried out at low speed. The test was repeated at $F_{h}$ is 0.33 at zero drift angle which is considered a very low speed. Therefore, type uncertainty B will be high for this particular case. Elsherbiny et al. [16] justified that at low speeds, the squat is always recorded in the range of few millimeters. Since type A uncertainty is the dominant value in the total uncertainty, and is independent of the ship speed, the percentage uncertainty for low speeds will be large when compared to the uncertainty at high speeds. This happens due to instrument resolution, where the minimum measurable sinkage is similar to the ship sinkage for the low speed tests. For further discussion on the experimental uncertainty, obtained form these tests, the reader is referred to Elsherbiny et al. [26].

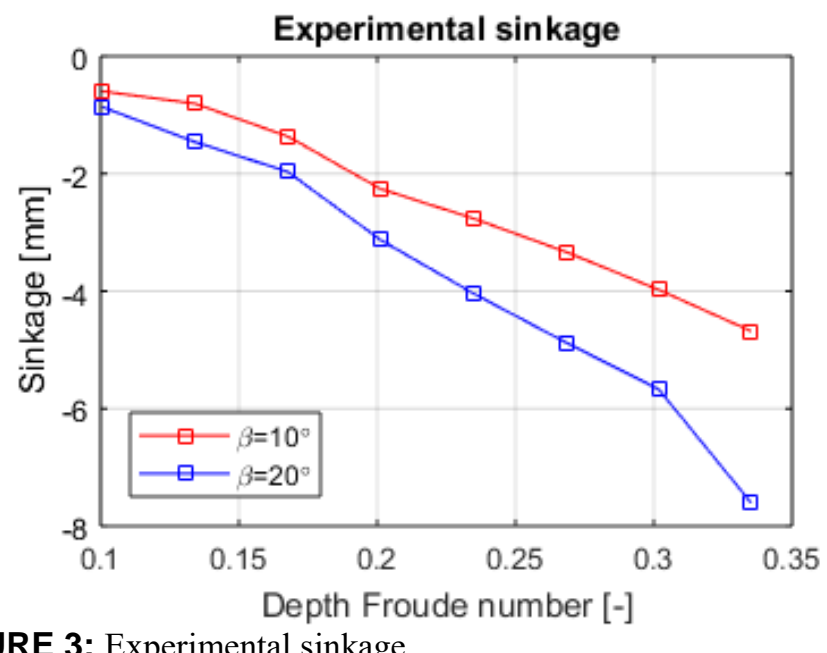

FIGURE 3: Experimental sinkage

The experimentally obtained sinkage is shown in Figure 3, whereas the trim is depicted in Figure 4 for both drift angles $(\beta=$ $10^{\circ}$ and $\left.\beta=20^{\circ}\right)$. The total force, drag force, lift force, and moment coefficients are shown in Figures 5-8. 


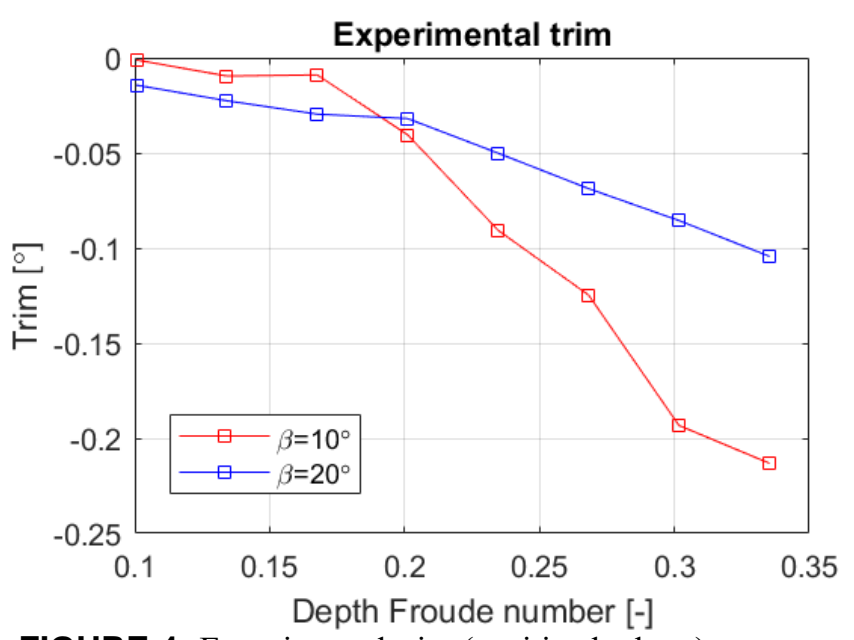

FIGURE 4: Experimental trim (positive by bow).

Experimental $\mathbf{C}_{\mathbf{T}}$

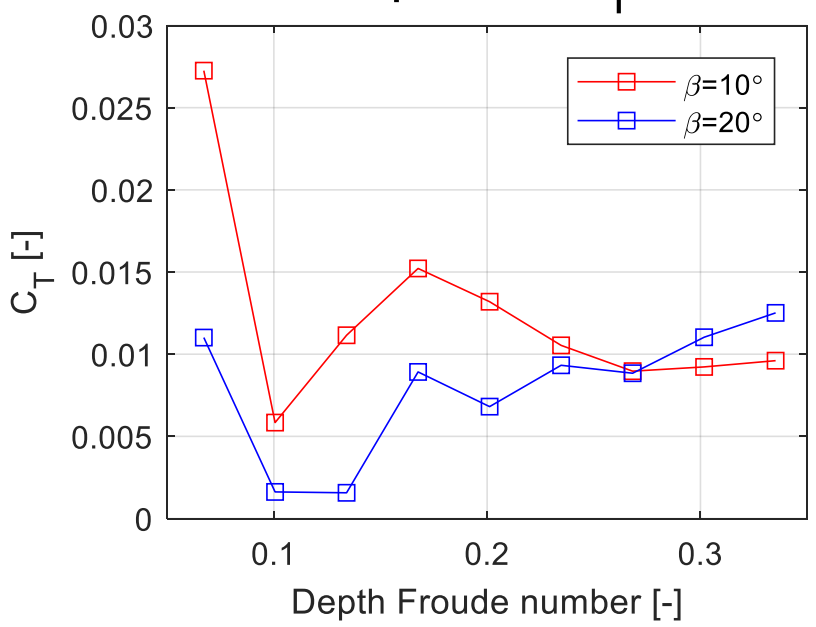

FIGURE 5: Total force coefficient.

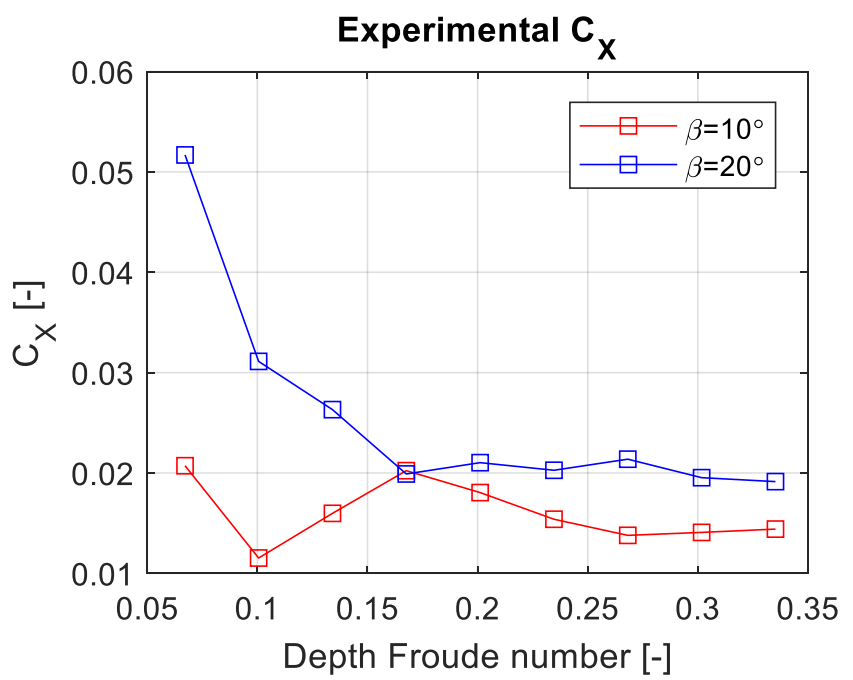

FIGURE 6: Drag force coefficient.

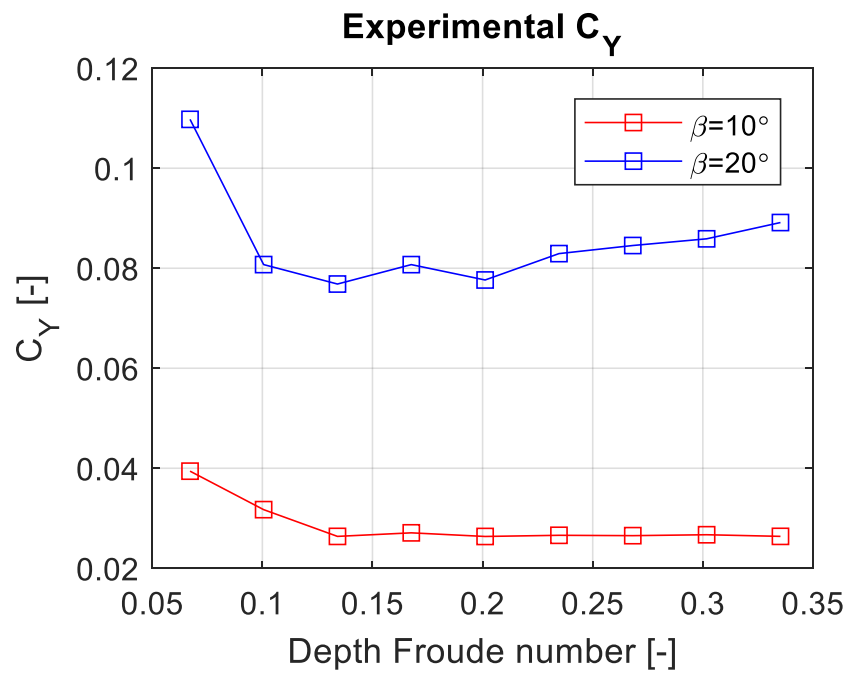

FIGURE 7: Lift force coefficient.

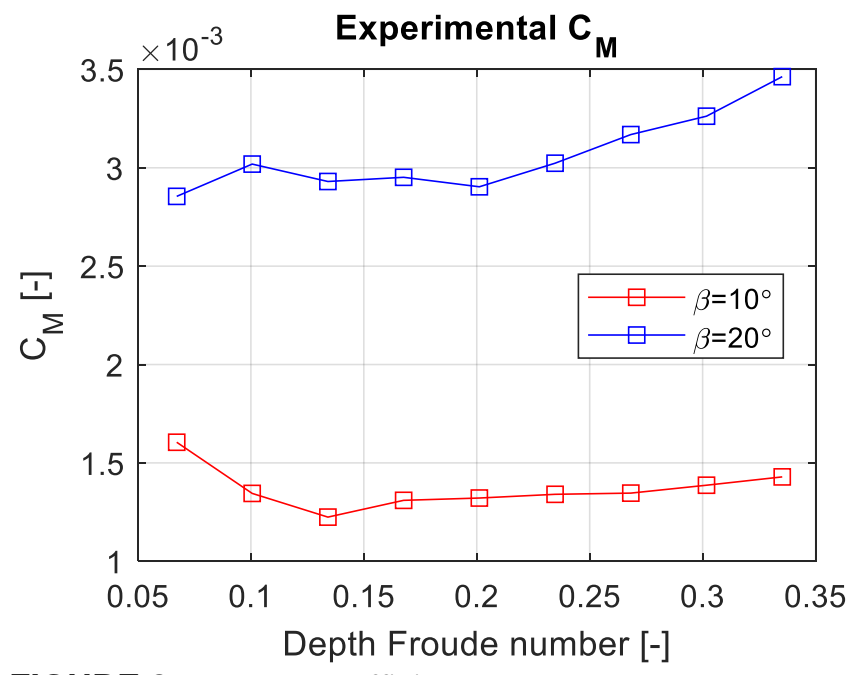

FIGURE 8: Moment coefficient.

Figures 3-5 clearly demonstrate that a varying drift angle has a pronounced effect on all examined parameters. At $\beta=20^{\circ}$, the ship sinkage and trim changes in an approximately linear fashion. On the other hand, at $\beta=10^{\circ}$, the abovementioned metrics vary quadratically, as is typically the case. This difference can be explained as being a direct consequence of the large drift angle. Therefore, with drift angle, the ship's sinkage and trim are impacted considerably. This is likely caused by the different flow properties, encountered at each drift angle. Such an explanation also accounts for the different behavior of the curves shown in Figures 5-8. Not surprisingly, the greatest differences between the two drift angles are seen in terms of lift force and hydrodynamic moment. Both metrics are several times higher in $\beta=20^{\circ}$, than in $\beta=10^{\circ}$.

\section{Numerical modelling and implementation}

This section presents the details pertaining to the numerical modelling approach and implementation. 
The solver, employed in this work, Star-CCM+, version 13.06, uses the finite volume method to discretize the domain into a set of adjoining cells. Continuity and momentum are linked via a predictor-corrector approach [22]. Although turbulence modelling forms a major part of this work, the details related to each model are not presented here. Instead, the reader is referred to the relevant literature, where several authors have compiled comprehensive and exhaustive reviews [27]-[30]. As stated earlier, the turbulence models tested are limited to some of the popular choices of the field [31]. These include the $k-\epsilon$ model [17], [18], the $k-\omega$ model [19], the $k-\omega S S T$ model [20], and the one-equation SPAL model [21]. The 24 case-studies (12 for each drift angle) examined in this study are summarized in Table 4. The full-scale equivalent speed is also shown to put the conditions into context. In shallow waters, ships tend to operate at low speeds due to the risk of grounding. In cases where canals are involved, there are frequently legal restrictions. For example, the Suez Canal Authority does not allow ships to advance at a speed greater than 7 knots [32], whereas in the Panama Canal, authorities restrict the speed of vessels from 6 to 10 knots depending on the section of the canal and ship type [33]. Thus, the choice of speeds was made to deliberately reinforce the practical applicability of this study.

Table 4. Computational conditions

\begin{tabular}{|c|c|c|c|c|}
\hline $\begin{array}{l}\text { Drift } \\
\text { angle } \\
\beta\left[^{\circ}\right]\end{array}$ & $\begin{array}{l}\text { Turbulence } \\
\text { model }\end{array}$ & $U[\mathrm{~m} / \mathrm{s}]$ & $F_{h}$ & $\begin{array}{l}U[\mathrm{kn}] \text { (full-scale } \\
\text { equivalent) }\end{array}$ \\
\hline \multirow{12}{*}{$\begin{array}{c}10^{\circ} \text { and } \\
20^{\circ}\end{array}$} & \multirow[t]{3}{*}{$k-\epsilon$} & 0.297 & 0.168 & 5 \\
\hline & & 0.416 & 0.235 & 7 \\
\hline & & 0.594 & 0.335 & 10 \\
\hline & \multirow[t]{3}{*}{$k-\omega$} & 0.297 & 0.168 & 5 \\
\hline & & 0.416 & 0.235 & 7 \\
\hline & & 0.594 & 0.335 & 10 \\
\hline & \multirow[t]{3}{*}{$k-\omega S S T$} & 0.297 & 0.168 & 5 \\
\hline & & 0.416 & 0.235 & 7 \\
\hline & & 0.594 & 0.335 & 10 \\
\hline & \multirow[t]{3}{*}{$\overline{S P A L}$} & 0.297 & 0.168 & 5 \\
\hline & & 0.416 & 0.235 & 7 \\
\hline & & 0.594 & 0.335 & 10 \\
\hline
\end{tabular}

The selected turbulence models are coupled with a second order accurate upwind scheme applied to the convective term of the RANS equations.

In cases where the drift angle is large, i.e. where the ship is modelled at a $20^{\circ}$ angle to the incident flow, flow separation is likely, especially for the highest examined speed. This suggests that the use of wall functions to treat the near-wall flow is not desirable [34]. For this reason, the mesh near the hull is constructed using concentric volumetric refinements, terminating in a prism layer to guarantee that $y^{+}<1$ over the wetted area of the hull, as shown in Figure 9 for $\beta=20^{\circ}, F_{h}=0.335$. It should be noted that meshing was performed with the aid of the automated procedures offered by Star-CCM+. The generated mesh resulted in a cell count of approximately 4.62 million, and is shown in Figure 10 for the $20^{\circ}, F_{h}=0.335$ case.

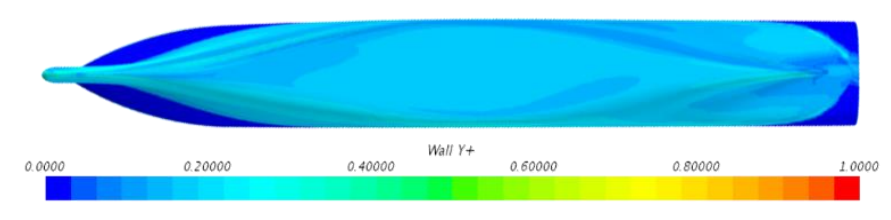

FIGURE 9: Bottom view of wall $y^{+}$for $\beta=20^{\circ}, F_{h}=0.335$.

As can be seen in Figure 10, the ship is rotated around the center of gravity with respect to the canal's centerline to model the selected condition. To simplify the case-studies, the hull's vertical position is adjusted in line with the experimentally determined sinkage and trim (Figure 3 and Figure 4), and kept fixed throughout the duration of the simulation. This reduces transient effects of the monitored integral quantities and thus speeds up convergence of the data.

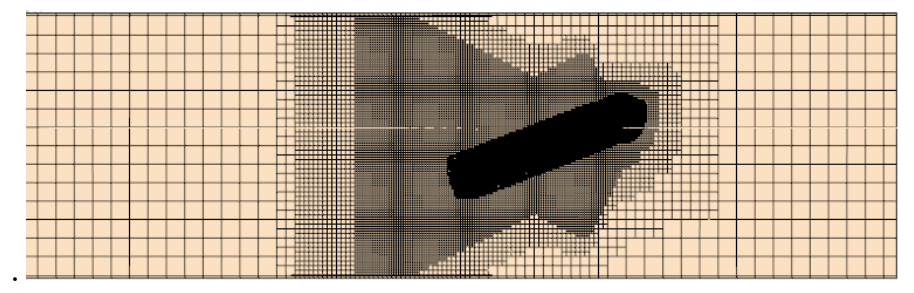

FIGURE 10: Top view of the generated computational mesh. Depicted: $\beta=20^{\circ}$.

To ensure that all parameters have converged sufficiently, each numerical simulation was allowed to progress a minimum 250 s of physical time. To model the temporal term in the governing equations, a first order scheme is used, with a time step of $0.0035 \mathrm{~L} / U$. This has previously been used to assess a variety of calm water cases and proved effective [35]. In any case, uncertainties and numerical errors due to the temporal term are typically of secondary importance, when compared to those induced by the choice of grid [36], which is assessed in the following section. It should be noted that the generated grid is maintained constant across the speed range. However, the volumetric refinements are adjusted to follow the orientation of the ship, resulting in near-identical cell counts across all cases.

Figure 10 also shows the extents of the computational domain. This extends 1.5 ship lengths upstream of the forward perpendicular, and 2.5 ship lengths downstream of the aft perpendicular when the ship is at a $0^{\circ}$ drift angle (i.e. prior to rotating the ship). The seabed is modelled in line with the experimental condition of $0.32 \mathrm{~m}$ below the mean free-surface, whereas the domain top is located 1.25 ship lengths from the free-surface. Both of these boundaries along with the inlet are assigned as velocity inlets in the computational domain. The domain sides are slip walls, and are located a distance of $2.3 \mathrm{~m}$ from the canal centerline, in line with the experimental condition (4.6m total width). Finally, the domain outlet maintains the hydrostatic pressure via the pressure outlet condition. 
To model the selected speed, the concept of a flat wave is used, which assigns the corresponding current velocities to the air and water. The interphase between the two is captured via the Volume of Fluid method [37].

\section{Results and discussion}

This section contains the resulting data, generated in the course of the numerical study. The assessed integral quantities are depicted alongside their numerical uncertainty as well as the experimental value. The former is assessed via the procedures endorsed by the International Towing Tank Conference (ITTC, [38]). In the interest of brevity, the relationships used to produce estimates are omitted. These can be accessed in the open literature [39]. However, it is important to mention that a refinement ratio of $\sqrt{2}$ was used to produce three geometrically similar grids. These featured $4.62 \mathrm{mil}, 2.72 \mathrm{mil}$, and $1.51 \mathrm{mil}$ cells for the fine, medium, and coarse grids, respectively. To enable an uncertainty assessment for cases where monotonic convergence was not observed, the modified relationships recommended by the ITTC were employed [38]. In any case, monotonic convergence and oscillatory convergence are the only modes in which the data changed as a result of grid refinement in all cases.

To begin with, the three force components are assessed. These are depicted in Figures 11-15. It should be noted that in all cases, the $x$ and $y$ components have been multiplied by their corresponding factor as shown in Eq. (1) to enable their aggregation and comparison with the total resistance coefficient. In the figures, the $y$ component and total force coefficient obtained experimentally are shown in an attempt to avoid overloading the figures. The $x$ component of the force may be deduced by the difference between the two. In all cases, the total force coefficient is predicted with reasonably good accuracy. In the $\beta=10^{\circ}$ cases, the $y$ component of the force is systematically underpredicted by all turbulence models. The numerical uncertainties, associated with each model increase with the ship speed. However, the accuracy level remains largely unchanged. In other words, a more or less constant margin of error is reproduced across the $\beta=10^{\circ}$ cases in terms of $\mathrm{C}_{\mathrm{Y}}$. The $x$ component of the force is seen as more problematic to predict by the numerical model. This conclusion can be arrived at based on Figures 10-13. Here, although the $y$ force predictions exhibit a systematic underprediction, the $x$ force is seen to compensate the total. Thus, $\mathrm{C}_{\mathrm{X}}$ is overpredicted across the lowest and highest speed for $\beta=10^{\circ}$, whereas for $F_{h}=0.235$, the $x$ force is predicted with good accuracy.

The SST turbulence model has a tendency to exhibit the largest numerical uncertainty of all two-equation models. However, the accuracy of the model is not seen to be compromised at the grid density employed presently. The oneequation turbulence model provides predictions, broadly in line with the two-equation models. In $\beta=10^{\circ}, F_{h}=0.335$, the numerical uncertainty is seen to be highest for the $y$ force when using the SPAL closure. However, the overall performance of the model is demonstrated to be as adequate as the more complex two-equation family of models. The fact that the SPAL model requires less computational time due to the reduced number of additional equations used to close the governing equations makes it a good choice when the cell number is high. On the other hand, turbulence equations are known to scale well with increasing cell numbers [40], i.e. limiting its attractiveness in cases with very large cell counts. Coincidently, one might prefer to switch to scale resolving options to model turbulent properties. Recent research [41] suggests that the use of Detached Eddy Simulation (DES) can be highly beneficial in terms of accuracy without resorting to extremely high cell numbers, as would be the case if one opted for Large Eddy Simulation (LES) instead [42]. Such approaches have been applied to study the problem of ships advancing with a drift angle [11], [12], [43]. In this study, widely used models are used in favor of scale resolving alternatives.

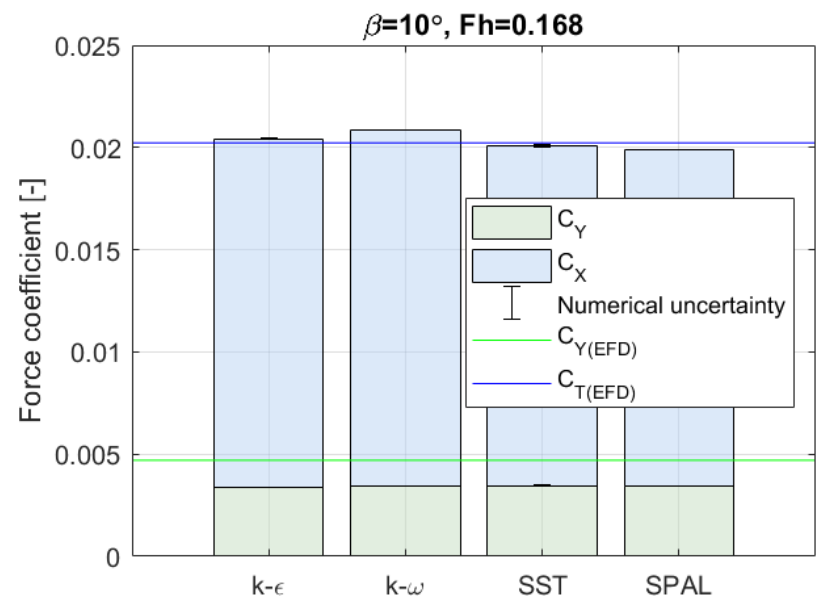

FIGURE 11: Numerical force coefficients comparison, $\beta=10^{\circ}, F_{h}=0.168$.

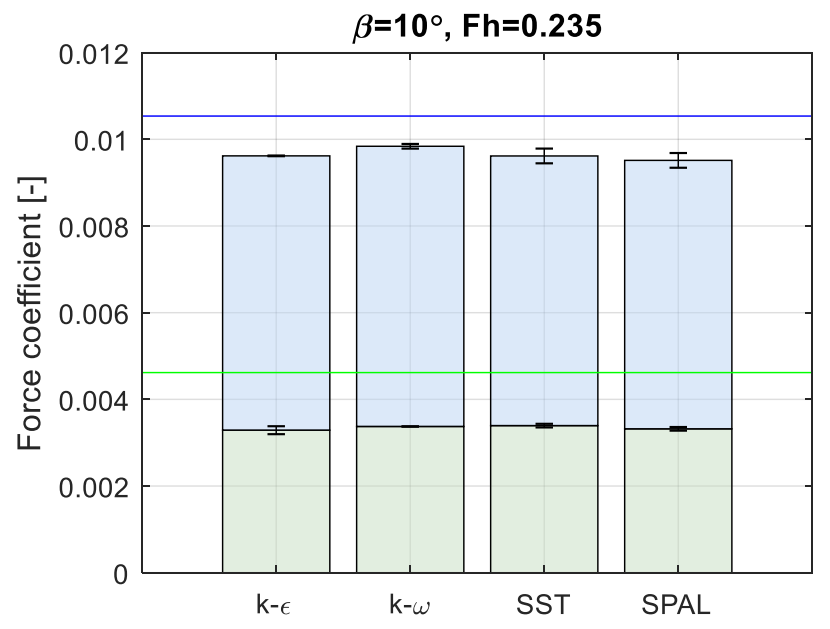

FIGURE 12: Numerical force coefficients comparison, $\beta=10^{\circ}, F_{h}=0.235$. The legend entries follow from Figure 11 . 


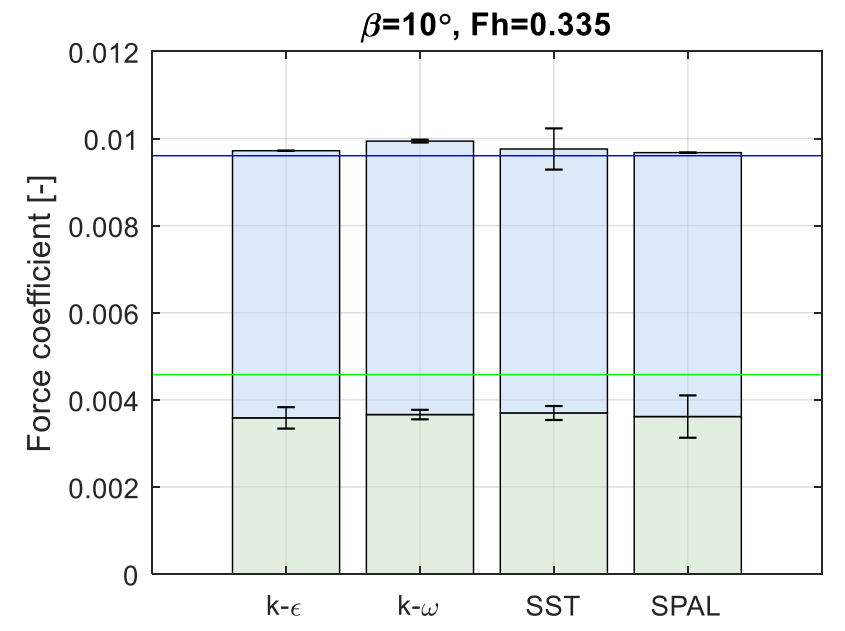

FIGURE 13: Numerical force coefficients comparison, $\beta=10^{\circ}, F_{h}=0.335$. The legend entries follow from Figure 11 .

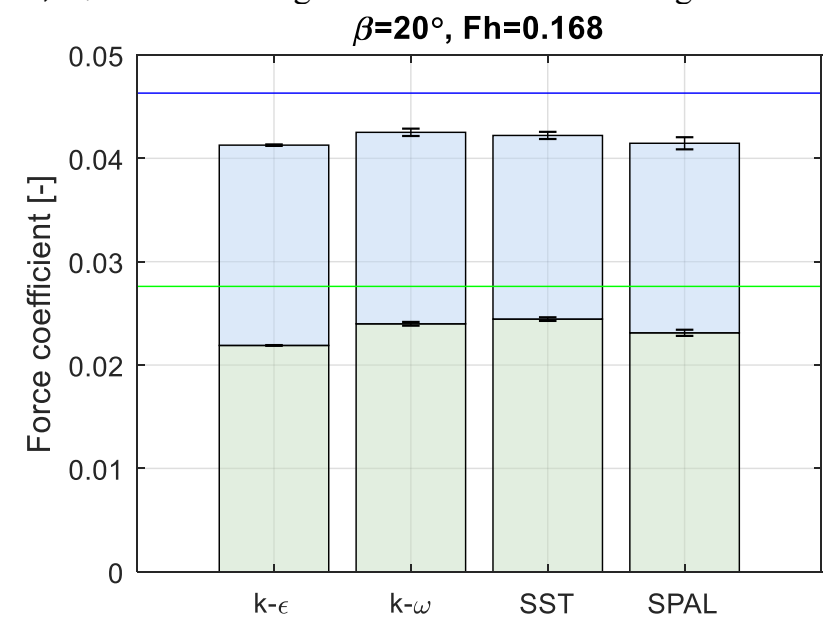

FIGURE 14: Numerical force coefficients comparison, $\beta=20^{\circ}, F_{h}=0.168$. The legend entries follow from Figure 11.

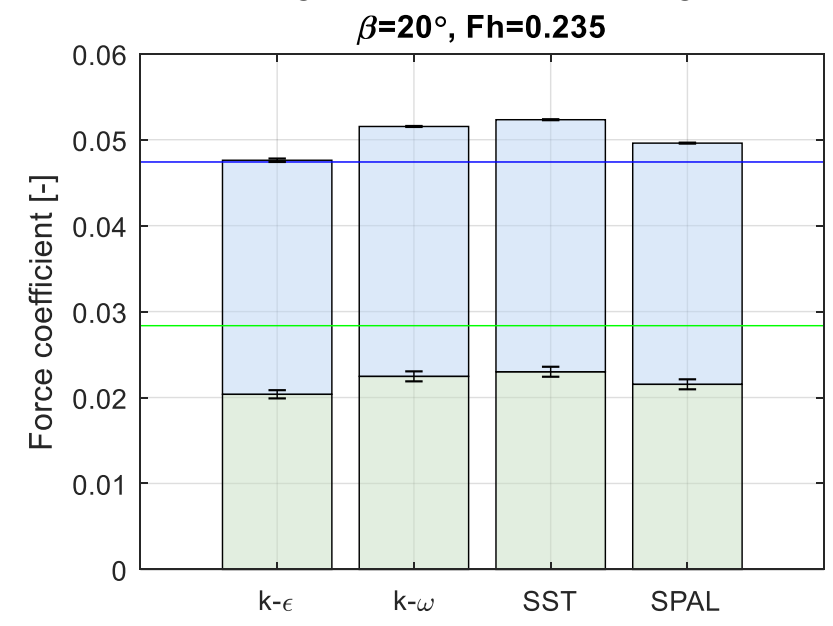

FIGURE 15: Numerical force coefficients comparison, $\beta=20^{\circ}, F_{h}=0.235$. The legend entries follow from Figure 11 .

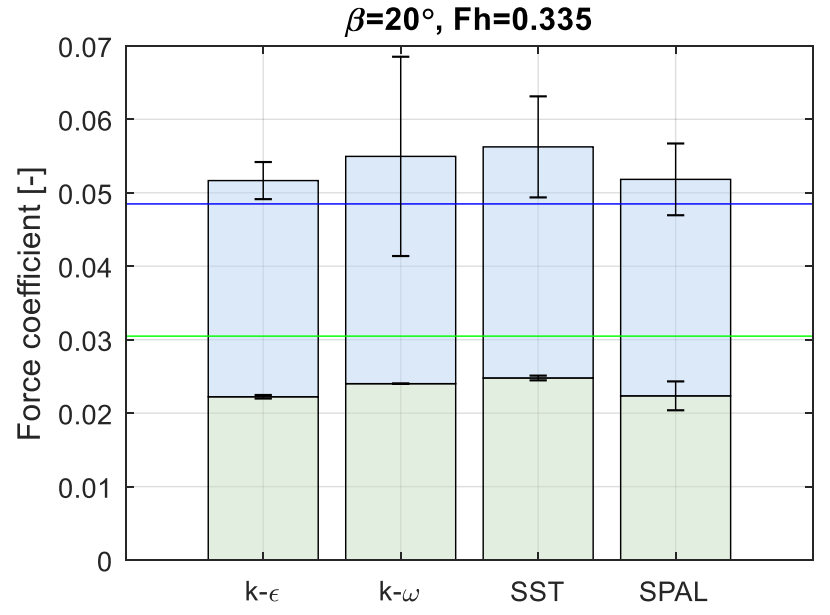

FIGURE 16: Numerical force coefficients comparison, $\beta=20^{\circ}, F_{h}=0.335$. The legend entries follow from Figure 11 .

Figures 14-16 depict the cases where the drift angle was set to $20^{\circ}$. As was the case for $\beta=10^{\circ}$, the $y$ component of the force is underpredicted by a more or less constant amount. On the other hand, the $x$ component of the force shifts from being in agreement with the experimental data to overpredicting it with increasing speed. The influence of the turbulence model also becomes more pronounced. Although this occurs simultaneously in both components of the total, $\mathrm{C}_{\mathrm{X}}$ exhibits greater variation with increasing speed. The numerical uncertainty in $C_{Y}$ is maintained low throughout the examined case-studies.

As can be expected, the numerical simulations become more challenging with increasing speed at $\beta=20^{\circ}$. This is evident by the increased uncertainty, as well as the disagreement with the experimental data. In reality, a condition where a ship drifts at $20^{\circ}$ with a speed of 10 knots is highly unlikely. However, it is a useful numerical experiment to show the performance of different models.

The assessment performed herein suggests that turbulence models behave similarly in all conditions except $\beta=20^{\circ}$, $F_{h}=0.335$. This is in line with findings of researchers examining resistance of ships at $\beta=0^{\circ}$. To elaborate, research has shown that calm water resistance prediction can be achieved reasonably well by most two-equation turbulence models, as well as the SPAL model [44]. The results presented herein suggest this also applies to drift conditions. It is more likely that grid topology and density have a greater influence on the integral parameters of interest than the turbulence model itself.

Typically, research examining drift angle conditions includes a discussion on the generated vortices in the wake of the ship. This is the case because the structure of vortices is highly dependednt on the turbulence model. Moreover, when a ship is simulated under a drift angle, the flow structures are more clearly discernible than at $\beta=0^{\circ}$. An example of the vortices generated is shown in Figure 17 for $\beta=20^{\circ}, F_{h}=0.335$ using all turbulence models. Here, three main vortex systems can be identified. One, shed from the bow and being detached into the free stream at the end of the bulbous bow. The second also originates from the bow, 
but remains attached over approximately half of the ship length, following which it is detached into the free stream. The third vortex is detached from the stern and is shed into the free stream. Previous research has shown that at higher drift angles $\left(30^{\circ}\right)$, the vortices shed from the bow may curve sufficiently, in effect interacting directly with the stern system of vortices [45]. Figure 16 suggests that this may occur in this case as well if the vortex were to be traced further downstream. In any case, this would occur at a significant distance aft of the ship and is not forecast to impact the ship substantially. The differences in choice of turbulence model as they relate to the formation and propagation of vortices is also evident based on their shape and dynamic pressure in Figure 16. The particular case of $\beta=20^{\circ}$ is chosen as it highlights the vortex structure the most. An in-depth examination of the vortex structure for each case and comparison between different turbulence models will form part of a wider study, which will supplement the present computations.

Next, the hydrodynamic moment predictions are assessed. These are shown for all turbulence models in Figure 18. As before, the experimental data alongside the numerical uncertainty is incorporated within the plot for clarity. For $\beta=10^{\circ}$, the $y$ limits of the axis has been maintained constant to enable a comparison with increasing speed. For $\beta=20^{\circ}, F_{h}=0.335$ this was breached due to the large numerical uncertainty associated with the prediction of the $k-\omega$ model. However, all models, except the $k-\epsilon$ exhibited this behavior. This is not surprising considering the large drift angle case to which the models are applied to, which makes computations challenging.

Figure 18 suggests that all moment coefficients are overpredicted. In the case where $\beta=10^{\circ}$, the numerical predictions increase at a rate faster than the experimentally determined moment coefficient. This results in an increase of the observed disagreement, although all turbulence models approximate the experimentally determined values well. In the case of $\beta=20^{\circ}$, a similar behavior of the numerical predictions is observed. The variability of the predictions across different turbulence models is not as great as was previously observed for the force coefficients. This is the case because the normal component of the resistance takes the dominant role in the hydrodynamic moment. Frictional effects, which are strongly dependent on the turbulence model are of lesser consequence. The reason why some difference is observed relates to the cascade of turbulent quantities into the wake and the region near the ship. These affect the pressure field near the ship, thus generating a different force distribution on the hull. A first indication of this effect can be observed in Figure 17 in terms of dynamic pressure distribution of the vortex structure in the wake.

\section{Conclusion}

This work has presented an experimental and numerical study on the performance of the KCS at two drift angles in a restricted water case. The experimentally determined forces and moments were compared against four widely used turbulence models. These demonstrated that turbulence modelling becomes a significant factor only at high speeds and drift angles, which are unlikely to occur in practice. Nevertheless, such cases present a useful field of study because they highlight the different performance of each model. The discrepancy in predicted forces and moments was shown to vary with increasing speed and drift angle.

As part of this study, the grid uncertainty was estimated for all case-studies. This proved to be of varying importance depending on the case. For instance, the highest examined speed showed low grid dependence for $\beta=10^{\circ}$, but high sensitivity to the mesh at $\beta=20^{\circ}$. An assessment of the choice of time step $(0.0035 \mathrm{~L} / U)$ should also be carried out in a similar fashion. This was not performed as part of this study because of the large number of additional simulations required (an additional 48 simulations are necessary to create the medium and coarse solutions). This is left as a piece of future work, which will form part of a larger study. A local examination of the generated vortices and more in-depth comparison between the different turbulence models is also warranted.

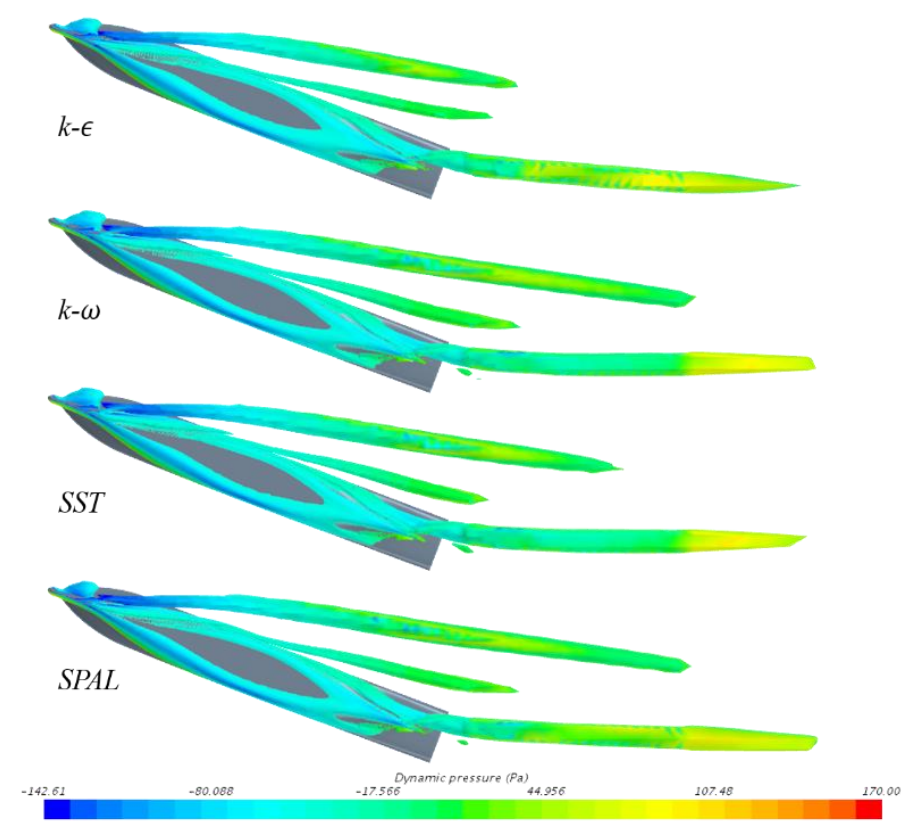

FIGURE 17: Bottom view of the generated vortices for $\beta=20^{\circ}$, $F_{h}=0.335, Q$-criterion $=1$. Colored with dynamic pressure.

\section{ACKNOWLEDGEMENTS}

Results were obtained using the ARCHIE-WeSt High Performance Computer (www.archie-west.ac.uk) based at the University of Strathclyde. The work reported in this paper is drawn from the first author's PhD thesis. The first author gratefully acknowledges the scholarship provided by the Faculty of Engineering at the University of Strathclyde, which fully supports his PhD. 

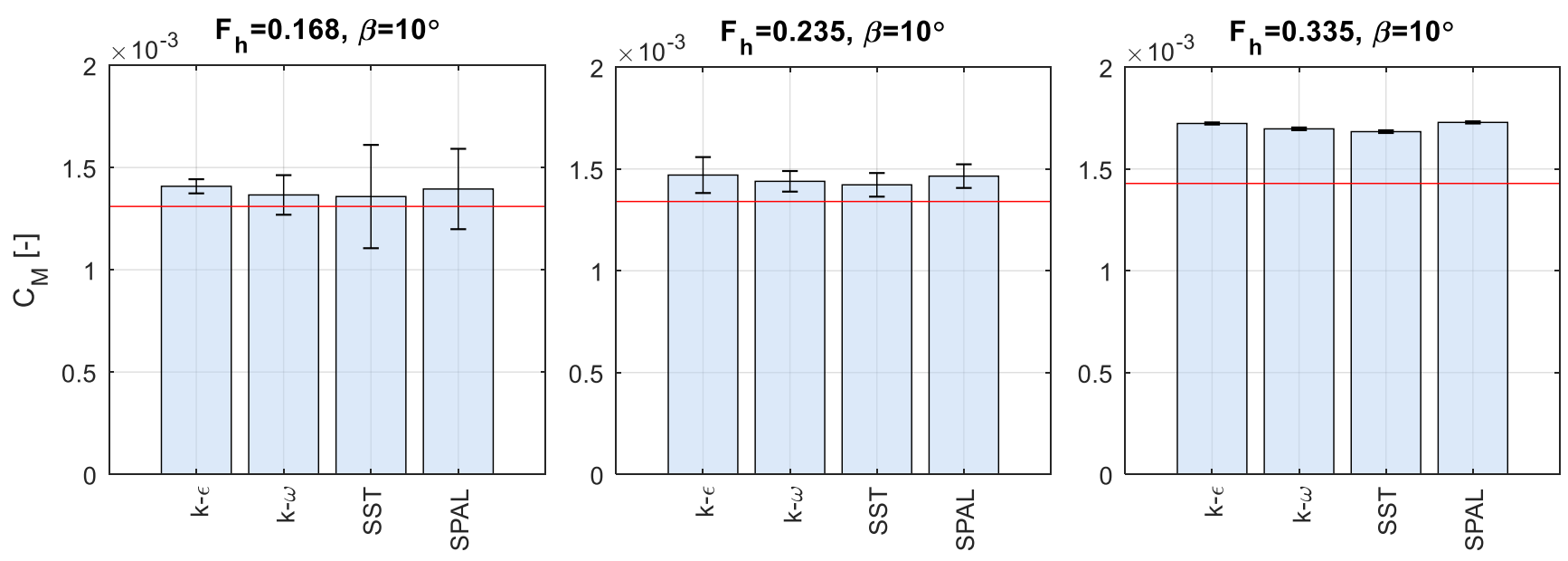
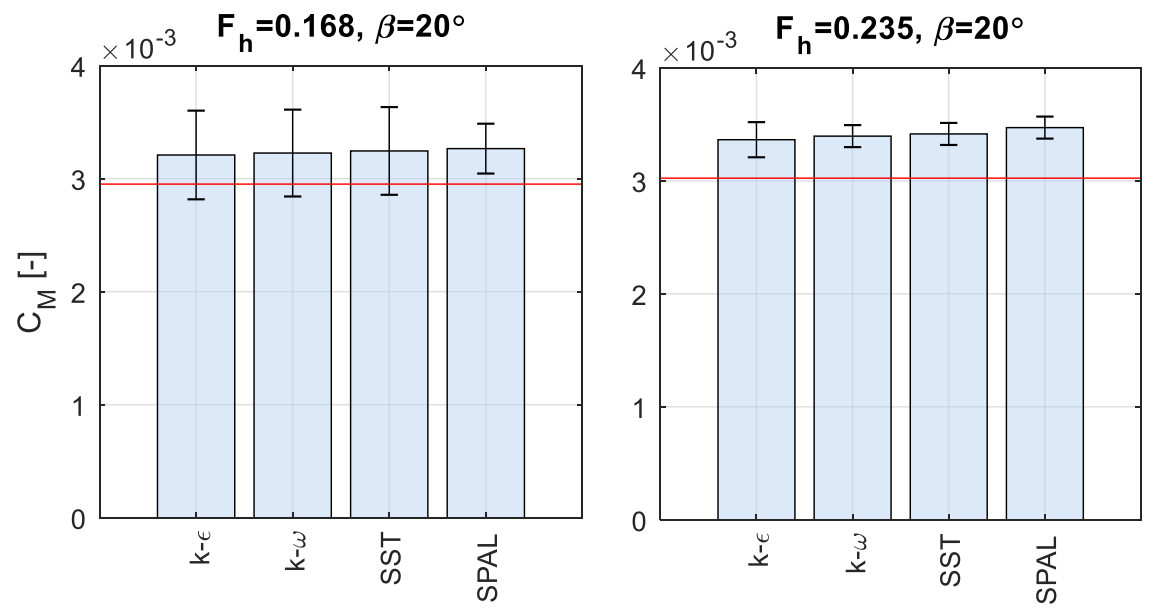

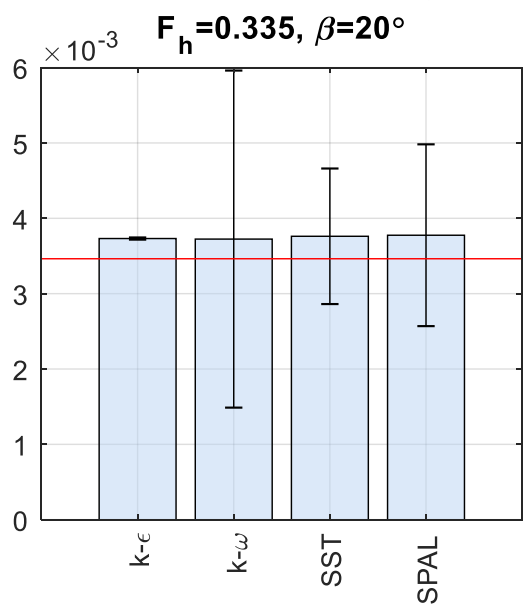

FIGURE 18: Moment coefficient for all cases.

[7] P. Mucha, T. Dettmann, V. Ferrari, and O. el Moctar, "Experimental investigation of free-running ship manoeuvers under extreme shallow water conditions," Appl. Ocean Res., vol. 83, no. September 2018, pp. 155162, 2019.

\section{REFERENCES}

[1] M. Terziev, T. Tezdogan, E. Oguz, T. Gourlay, Y. K. Demirel, and A. Incecik, "Numerical investigation of the behaviour and performance of ships advancing through restricted shallow waters," J. Fluids Struct., vol. 76, pp. 185-215, 2018.

[2] T. Tezdogan, A. Incecik, and O. Turan, "A numerical investigation of the squat and resistance of ships advancing through a canal using CFD," J. Mar. Sci. Technol., vol. 21, no. 1, pp. 86-101, 2016.

[3] P. Mucha and B. el Moctar, "Numerical Prediction of Resistance and Squat for a Containership in Shallow Water," no. May 2015, 2014.

[4] P. Mucha, O. el Moctar, T. Dettmann, and M. Tenzer, "An experimental study on the effect of confined water on resistance and propulsion of an inland waterway ship," Ocean Eng., vol. 167, no. March, pp. 11-22, 2018.

[5] P. Mucha, "On Simulation-based Ship Maneuvering Prediction in Deep and Shallow Water," 2017.

[6] P. Mucha, O. el Moctar, T. Dettmann, and M. Tenzer, "Inland waterway ship test case for resistance and propulsion prediction in shallow water," Sh. Technol. Res., vol. 64, no. 2, pp. 106-113, 2017.
[8] P. Mucha, G. Deng, T. Gourlay, and O. el Moctar, "Validation studies on numerical prediction of ship squat and resistance in shallow water," Proc. 4th MASHCON, no. May, pp. 83-92, 2016.

[9] Q. J. Meng and D. C. Wan, "Numerical simulations of viscous flow around the obliquely towed KVLCC2M model in deep and shallow water," J. Hydrodyn., vol. 28, no. 3, pp. 506-518, 2016.

[10] S. Lee and C. Hong, "Study on the course stability of very large vessels in shallow water using CFD," Ocean Eng., vol. 145, no. January, pp. 395-405, 2017.

[11] N. Abbas and N. Kornev, "Validation of hybrid URANS/LES methods for determination of forces and wake parameters of KVLCC2 tanker at manoeuvring conditions," Sh. Technol. Res., vol. 63, no. 2, pp. 96-109, 2016.

[12] C. Fureby, S. L. Toxopeus, M. Johansson, M. Tormalm, and K. Petterson, "A computational study of the flow around the KVLCC2 model hull at straight ahead conditions and at drift," Ocean Eng., vol. 118, pp. 1-16, 
2016.

[13] I. Razgallah, S. Kaidi, H. Smaoui, and P. Sergent, "The impact of free surface modelling on hydrodynamic forces for ship navigating in inland waterways: water depth, drift angle, and ship speed effect," J. Mar. Sci. Technol., pp. 1-22, 2018.

[14] J. P. Hooft, "Manoeuvring Large Ships in Shallow Water - II," 2018.

[15] K. Elsherbiny, T. Tezdogan, M. Kotb, A. Incecik, and A. H. Day, "An experimental investigation of the trim effect on the behaviour of a containership in shallow water," in Proceedings of the ASME 2019 38th International Conference on Ocean, Offshore and Arctic Engineering OMAE2019, Glasgow, UK, 2019.

[16] K. Elsherbiny, T. Tezdogan, M. Kotb, A. Incecik, and S. Day, "Experimental analysis of the squat of ships advancing through the New Suez Canal," Ocean Eng., vol. 178, no. November 2018, pp. 331-344, 2019.

[17] W. P. Jones and B. E. Launder, "The Prediction of Laminarization with a Two-Equation Model of Turbulence," Int. J. Heat Mass Transf., vol. 15, pp. 301314, 1972.

[18] B. E. Launder and B. I. Sharma, "Application of the energy-dissipation model of turbulence to the calculation of flow near a spinning disc," Lett. Heat Mass Transf., vol. 1, no. 2, pp. 131-137, 1974.

[19] D. C. Wilcox, "Formulation of the k-w Turbulence Model Revisited," AIAA J., vol. 46, no. 11, pp. 28232838, 2008.

[20] F. R. Menter, "Two-equation eddy-viscosity turbulence models for engineering applications," AIAA J., vol. 32, no. 8, pp. 1598-1605, 1994.

[21] P. Spalart and S. Allmaras, "A one-equation turbulence model for aerodynamic flows," 30th Aerosp. Sci. Meet. Exhib., 1992.

[22] Siemens, "Star-CCM+ User Guide version 13.04." 2018.

[23] J. Longo and F. Stern, "Effects of drift angle on model ship flow," Exp. Fluids, vol. 32, no. 5, pp. 558-569, 2002.

[24] ITTC, "Example for Uncertainty Analysis of Resistance Tests in Towing Tanks," 2014.

[25] ISO GUM, "Guide to the Expression of Uncertainty in Measurement,(1995), with Supplement 1, Evaluation of measurement data," Organ. Stand. Geneva, Switzerland. JCGM 101, 2008.

[26] K. Elsherbiny, M. Terziev, T. Tezdogan, A. Incecik, and M. Kotb, "Numerical and experimental study on hydrodynamic performance of ships advancing through different canals," Ocean Eng., 2019.

[27] C. Roy and F. G. Blottner, "Assessment of One- and Two-Equation Turbulence Models for Hypersonic Transitional Flows," J. Spacecr. Rockets, vol. 38, no. 5, pp. 699-710, 2001.

[28] C. Roy and F. G. Blottner, "Review and Assessment of Turbulence Models for Hypersonic Flows: 2D/Asymmetric Cases," 44th AIAA Aerosp. Sci. Meet.
Exhib., no. January, 2006.

[29] D. C. Wilcox, Turbulence modeling for CFD, 3rd ed. DCW Industries, 2006.

[30] P. A. Durbin and B. A. Pettersson Reif, Statistical theory and modelling for turbulent flow, Second Edi., no. 2. Wiley, 2011.

[31] M. Terziev, T. Tezdogan, and A. Incecik, "Application of eddy-viscosity turbulence models to problems in ship hydrodynamics," Ships Offshore Struct., pp. 1-24, Aug. 2019.

[32] Suez Canal Authority, "Suez Canal Rules of Navigation," 2019.

[33] Panama Canal Authority, "Panama Canal Rules of Navigation," 2015.

[34] P. A. Durbin, "Near-wall turbulence closure modeling without 'damping functions," Theor. Comput. Fluid Dyn., vol. 3, no. 1, pp. 1-13, 1991.

[35] T. Tezdogan, A. Incecik, and O. Turan, "Full-scale unsteady RANS simulations of vertical ship motions in shallow water," Ocean Eng., vol. 123, pp. 131-145, 2016.

[36] L. Eca and M. Hoekstra, "Evaluation of numerical error estimation based on grid refinement studies with the method of the manufactured solutions," Comput. Fluids, vol. 38, no. 8, pp. 1580-1591, 2009.

[37] C. . W. Hirt and B. . D. Nichols, "Volume of fluid (VOF) method for the dynamics of free boundaries," J. Comput. Phys., vol. 39, no. 1, pp. 201-225, Jan. 1981.

[38] ITTC, "Uncertainty Analysis in CFD Verification and Validation Methodology and Procedures," 25th ITTC 2008, Resist. Comm., p. 12, 2008.

[39] G. K. Saha, K. Suzuki, and H. Kai, "Hydrodynamic optimization of ship hull forms in shallow water," J. Mar. Sci. Technol., vol. 9, no. 2, pp. 51-62, 2004.

[40] J. Hawkes, G. Vaz, A. B. Phillips, S. J. Cox, and S. R. Turnock, "On the strong scalability of maritime CFD," J. Mar. Sci. Technol., vol. 23, no. 1, pp. 81-93, 2018.

[41] B. Pena, E. Muk-Pavic, and D. Ponkratov, "Achieving a high accuracy numerical simulations of the flow around a full scale ship," in Proceedings of the International Conference on Offshore Mechanics and Arctic Engineering - OMAE, 2019, vol. 7A, no. 5, pp. 1-10.

[42] M. Liefvendahl and C. Fureby, "Grid requirements for LES of ship hydrodynamics in model and full scale," Ocean Eng., vol. 143, no. March, pp. 259-268, 2017.

[43] N. Abbas and N. Kornev, "Study of unsteady loadings on the propeller under steady drift and yaw motion using URANS, hybrid (URANS-LES) and LES methods," Sh. Technol. Res., vol. 63, no. 2, pp. 121-131, 2016.

[44] L. Larsson, F. Stern, and M. Visonneau, Numerical Ship Hydrodynamics: An assessment of the Gothenburg 2010 Workshop. Springer, 2014.

[45] T. Xing, S. Bhushan, and F. Stern, "Vortical and turbulent structures for KVLCC2 at drift angle 0,12 , and 30 degrees," Ocean Eng., vol. 55, pp. 23-43, 2012. 